\title{
Comparison of the Influence of New Generation Threads P(LA/ CL) HA And P(LA/CL) on Convalescence and Clinical Effects in Patients Undergoing Minimally Invasive Anti-Aging Treatments
}

\author{
Jendrysik $B^{1 *}$, Kadjaia $A^{2}$ and Sulamanidze $G^{2}$ \\ ${ }^{1}$ Veno-Med Medical Center, Poland \\ ${ }^{2}$ Total Charm Tbilisi, Georgia
}

Research article

Volume 5 Issue 1

Received Date: February 06, 2020

Published Date: February 27, 2020

*Corresponding author: Bożena Jendrysik, MD, General surgeon, Cosmetologist, Veno-Med Medical Center, Sokoła 25, 42-700 Lubliniec, Poland, Tel: +48 602790 688; +48 34356 3559;

Email: gabinet@veno-med.pl

\section{Abstract}

Early signs of aging of facial skin results from elastic tissue and collagen degradation, causing fine-to-deep wrinkles formation. Various modifications of the techniques have been introduced to fight against aging. In recent years, minimally invasive procedures, in particular tread lifting gained more and more interest both among patient and the practitioners. Nowadays, wide range of threads with different compositions and configurations are presented on the markets.

This pilot study was done to evaluate subjective assessments of $\mathrm{P}$ (LA/CL) (HA) and P (LA/CL) threads and compare their impact on convalescence and clinical outcome in patients undergoing various anti-aging treatments. Patients received appropriate non-invasive aesthetic treatment combined with P(LA/CL) HA and P(LA/CL) thread implantation on the opposing side of their face. The patients' subjective impressions, given as a numerical value based on the Global Aesthetic Improvement Scale, were collected on the day of the procedure and at 3,6, and 9 weeks after the procedure. The study involved 9 women, aged 40 to 63 , and investigated the assessment of the convalescence period, changes in the appearance and skin quality, as well as lifting and volumetric effects. The results revealed significant acceptance of the convalescence period, greater satisfaction with skin quality improvement, and similar lifting effects and volumetric changes.

Keywords: Face lifting; Threads; Hyaluronic acid; Skin texture

Abbreviations: P(LA/CA): Poly (Lactic Acid/ Caprolactone); HA: Hyaluronic Acid; TCA: Trichloracetic acid; RF: Radio Frequency; GAIS: Global Aesthetic Improvement Scale.

\section{Introduction}

Aging is a natural and common phenomenon [1-4]. It becomes evident in all face structures and leads to a progressive decreased loss of tissues, changes in the tension of retaining ligaments (laxity), and three-dimensional compartmentalization of the skin surface (sagging) [5].

Various complementary methods should be combined to achieve the desired therapeutic anti-aging effect on the skin and effectively slow the aging process. The symmetry and natural proportions of the face should also be retained.

Apart from well-known and widely used treatments such as the use of chemical peels, volumetric facelift, botulin toxin, 


\section{Clinical Dermatology Open Access Journal}

autologous lipotransfer (micro and nanofat) [6], and tissue remodeling $\left(\mathrm{CO}_{2}\right.$, needle and subcutaneous radiofrequency) [7], lifting threads [2], especially new-generation threads with hyaluronic acid, occupy an important place in the treatment of facial aging processes.

Lifting threads are a perfect solution for people who need treatments on an outpatient basis, which do not involve long recovery period, general anesthesia or scars. In some cases, age-related changes and tissue ptosis are so advanced that only plastic surgery is able to give the desired aesthetic effect. However, there is a group of patients in whose lifting threads can give a result comparable to a surgery. The best outcomes are obtained in patients with a small or medium degree of tissue drooping, loss of tissue volume, a sagging mid-facial appearance, prominence of the jaw, as well as in patients with some relapse from previous procedure such as facelift, facial asymmetry and skin dehydration. The threads are not suitable for patients with autoimmune diseases, blood disorder, anticoagulant intake, collagenosis, ischemic heart disease, inflammatory condition, susceptibility to keloid and hypertrophic scar formation, chronic diseases, pregnancy, earlier implanted non-absorbable threads or tumor in the to be treated area and idiosyncrasy to the thread composition.

The unique spatial design of the microscopic barbs allows a strong and stable lifting of soft tissues and obtaining volume through their reposition. An additional, important factor is the strong revitalizing effect of threads related to their chemical structure (polylactic acid, caprolactone, hyaluronic acid), which causes strong stimulation of collagen and elastin production, increased tissue hydration and reduction of the inflammatory reaction.

There has been performed a comparative clinical study to evaluate the effectiveness of implantation of P(LA/CL) HA and P(LA/CL) threads in the subcutaneous tissue of pigs. Histologic analysis was conducted on 14, 30, 60, 90 days after the procedure. The aim of this study is to evaluate the positive impact of the latest generation of Aptos threads with hyaluronic acid on the healing process and the final clinical effects based on patients own subjective observations. Factors such as patient comfort during the procedure, the acceptable course of convalescence, and long-lasting clinical effects that meet patients' expectations, both in terms of improving the skin quality as well as facelift and volumetry, should be considered before choosing the strategy individuals [8-12].

\section{Materials and Methods}

Nine patients, ages between 40-63, with exhibited typical signs of facial aging and were included in this study. The inclusion criteria were deterioration of skin quality and appearance, fat loss, contour disorder (furrows), and tissue dropping. Treatment approach varied in accordance with individual symptoms; mainly chemical peeling using trichloracetic acid (TCA), resurfacing of the skin with needle radio frequency (RF), collagen stimulating injections (sculptra), lipotransfer (microfat), and nanofat mesotherapy.

Treatments were combined with $\mathrm{P}(\mathrm{LA} / \mathrm{CL}) \mathrm{HA}$ and $\mathrm{P}(\mathrm{LA} / \mathrm{CL})$ thread implantation on opposing sides of the face. Patients' satisfaction was assessed in accordance with Global Aesthetic Improvement Scale (GAIS) Scale immediately after, 3,6 and 9 weeks after the procedure (Table No 1). The subjective assessment covered recovery time (pain, swelling, bruises), changes in the skin structure (elasticity, hydration, pigmentation, fine wrinkles, acne scars and pores), and lifting effects (soft tissue lifting, face contouring, volumetry).

The Global Aesthetic Improvement Scale was used to collect patient opinions (Table 1) and the results were presented in the form of diagrams (Figures 1-9.)

\begin{tabular}{|c|c|}
\hline Degree & Description \\
\hline 5 Exceptional improvement & Excellent corrective result after a session \\
\hline 4 Very improved & Marked improvement of the appearance, but not completely optimal \\
\hline 3 Improved & $\begin{array}{c}\text { Improvement of the appearance; better compared with the initial condition, but a touch-up is } \\
\text { advised }\end{array}$ \\
\hline 2 Unaltered & The appearance substantially remains the same as the original condition \\
\hline 1 Worsened & The appearance has worsened compared with the original condition \\
\hline
\end{tabular}

Table 1: The Global Aesthetic Improvement Scale.

\section{Results and Discussion}

Successful anti-aging results can be affected using various complementary methods. The course of treatment should be determined by the underlying conditions as well as the patient's comfort during the treatment and acceptable convalescence times. This study investigated the difference 
in patient reported satisfaction levels at time of treatment, and 3, 6, and 9 weeks after treatment using two differing types of Aptos threads, one with hyaluronic acid and one without.

The patients' subjective opinions emphasize that the hyaluronic acid contained in P(LA/CL) HA threads significantly affects the tissue regeneration processes in the postoperative period as well as accelerates and strengthens the effects of improving skin quality and appearance. However, no significant differences were observed in the final clinical effects of tissue lifting and volumetry. The individual patient results were dependent on prevailing clinical symptoms and treatment.

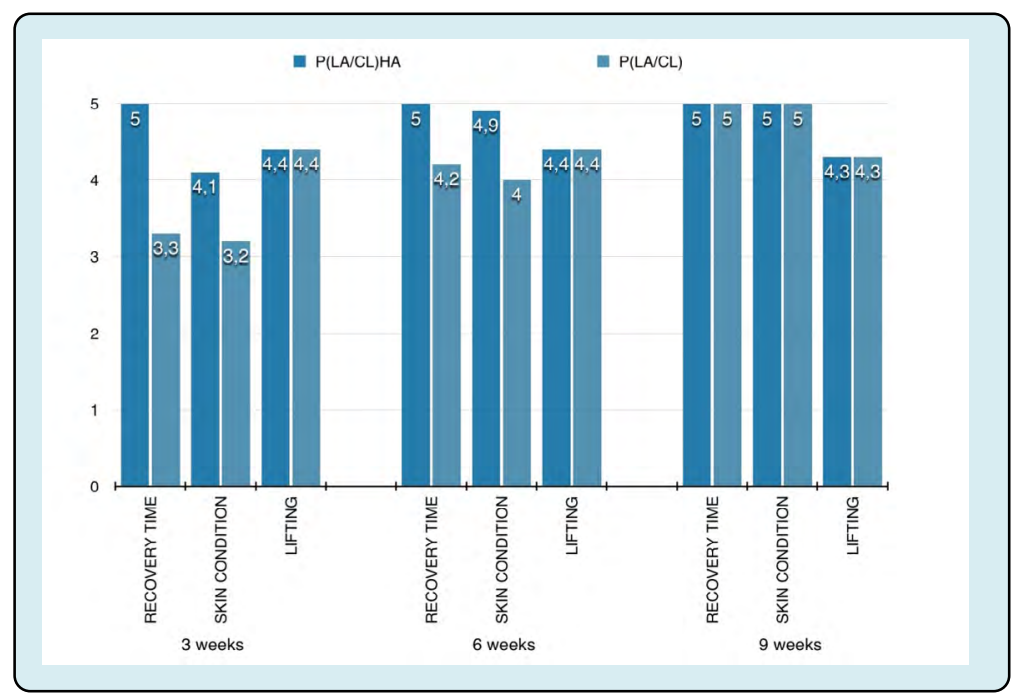

Figures 1-9 provide the clinical details on each of the individual cases.

\section{Clinical Cases}

\section{Patient 1}

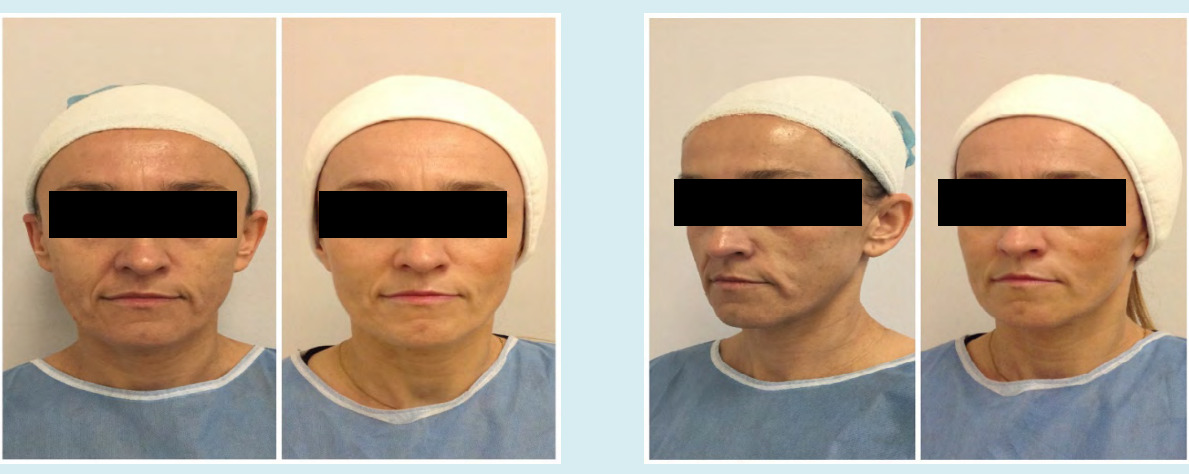

Figure 1: Patient age: 45.

Symptoms: Tired and sad facial expressions, drooping skin and marionette lines.

Treatment: Needle RF (2.5 mm) with P(LA/CL) and P(LA/ CL) HA implanted on opposing sides of the face.
Results: Soft tissue lifting in the middle part of the face with improved contour and marionettes lines. Improvement in skin quality, elasticity, skin resilience and the depth of the fine wrinkles. 


\section{Patient's Assessment}

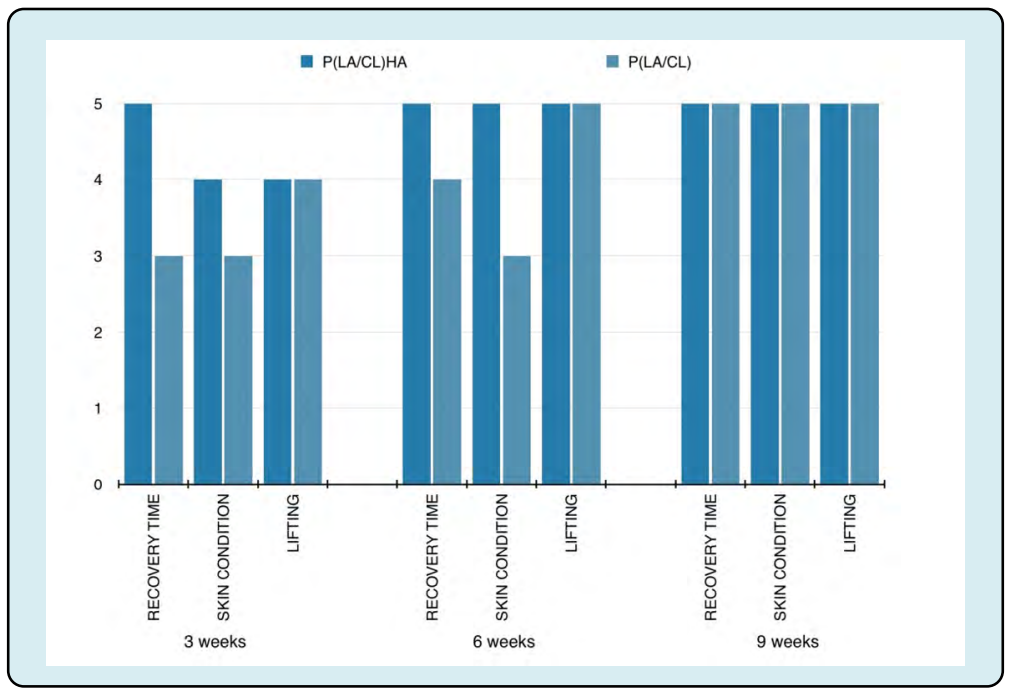

\section{Patient 2}

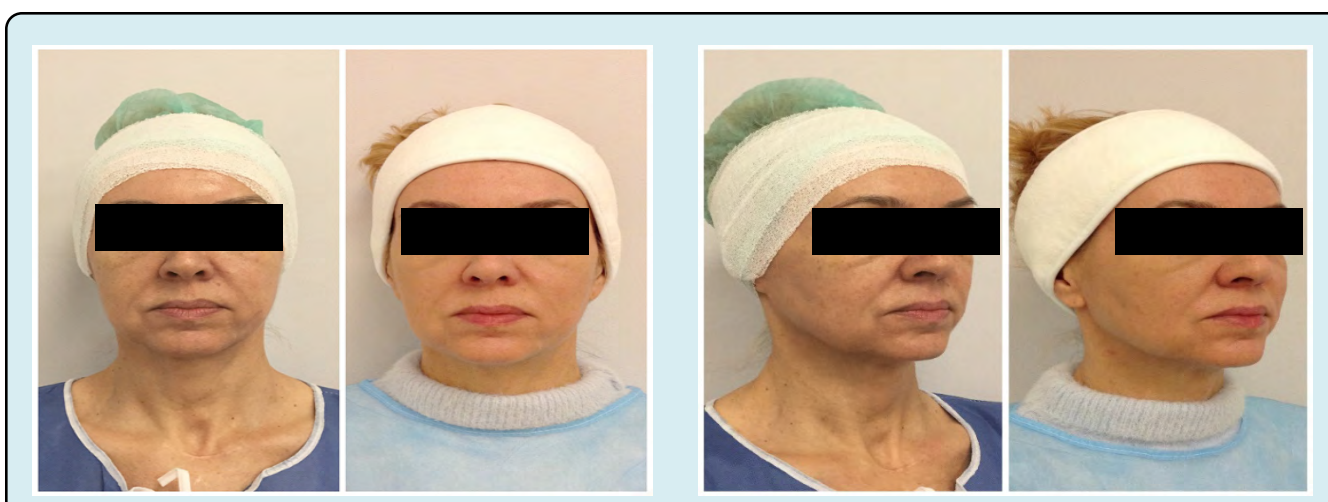

Figure 2: Patient age: 53.

Symptoms: Loss of skin elasticity and tension, wrinkles, minor post-traumatic and post-acne scars, as well as marked ptosis in the middle part of the face with lower eyelid edema. Treatment: Needle RF (2.5 mm) with simultaneous P(LA/
CL) and P(LA/CL) HA implantation.

Results: Improved skin density and contours and reduced wrinkles and appearance of acne scars and posttraumatic scars.

\section{Patient's Assessment}

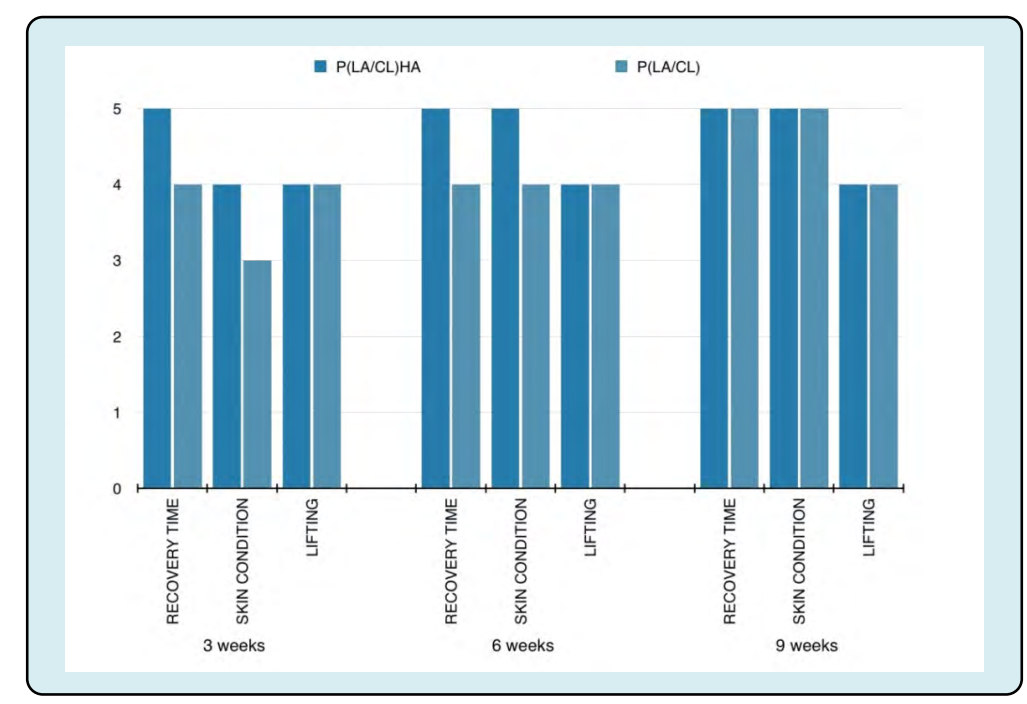




\section{Patient 3}
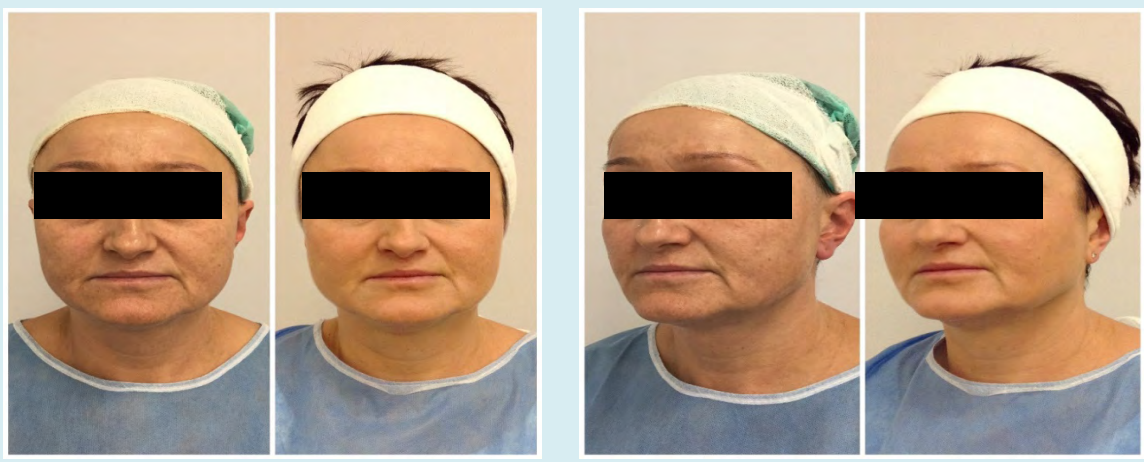

Figure 3: Patient age: 52.

Symptoms: Thick, heavy skin with enlarged pores and irregular hyperpigmentation. Atrophic acne scars and marked ptosis of the soft tissues in the middle and lower part of the face.

Treatment: P(LA/CL) and P(LA/CL) HA thread implantation with TCA peeling at the same time and at 4 weeks after thread implantation.

Results: Significant reduction in hyperpigmentation, softer skin and smaller pores. Reduction of nasolabial folds and marionettes and contour improvement.

\section{Patient's Assessment}

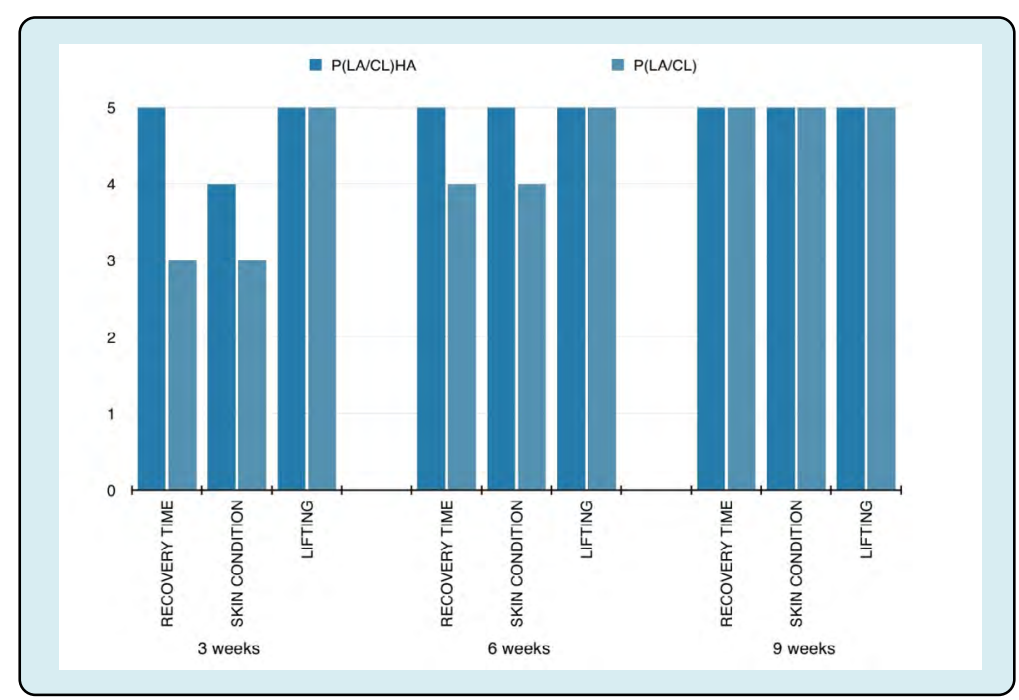

\section{Patient 4}

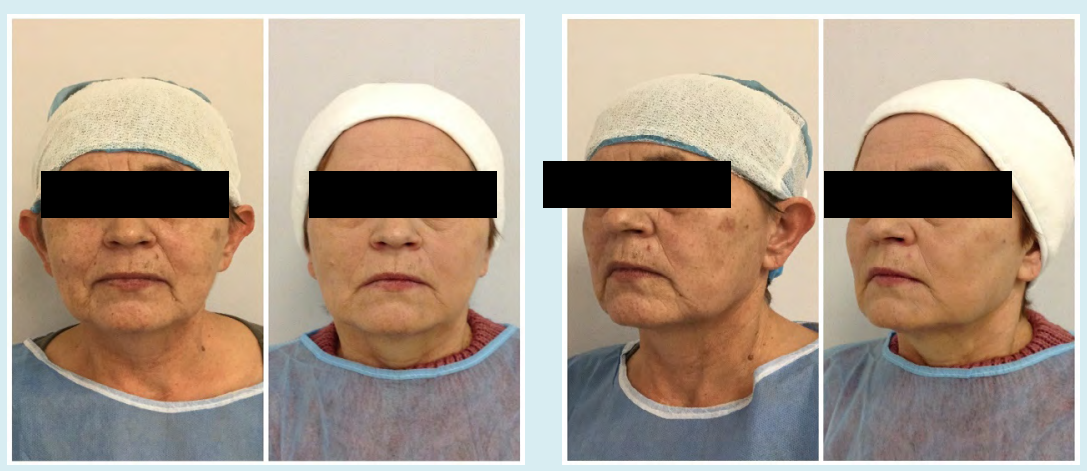

Figure 4: Patient age: 60. 
Symptoms: Soft tissue dropping in the middle and lower part of the face with visible nasolabial folds and marionette lines as well as flaccid skin with hyperpigmentation.

Treatment: P(LA/CL) and P(LA/CL) HA thread implantation with concurrent TCA peeling. TCA peeling was performed again 4 weeks after thread implantation.

Results: Significant reduction in hyperpigmentation, an improvement in skin density, flattening of wrinkles and noticeable lifting of soft tissues.

\section{Patient's Assessment}

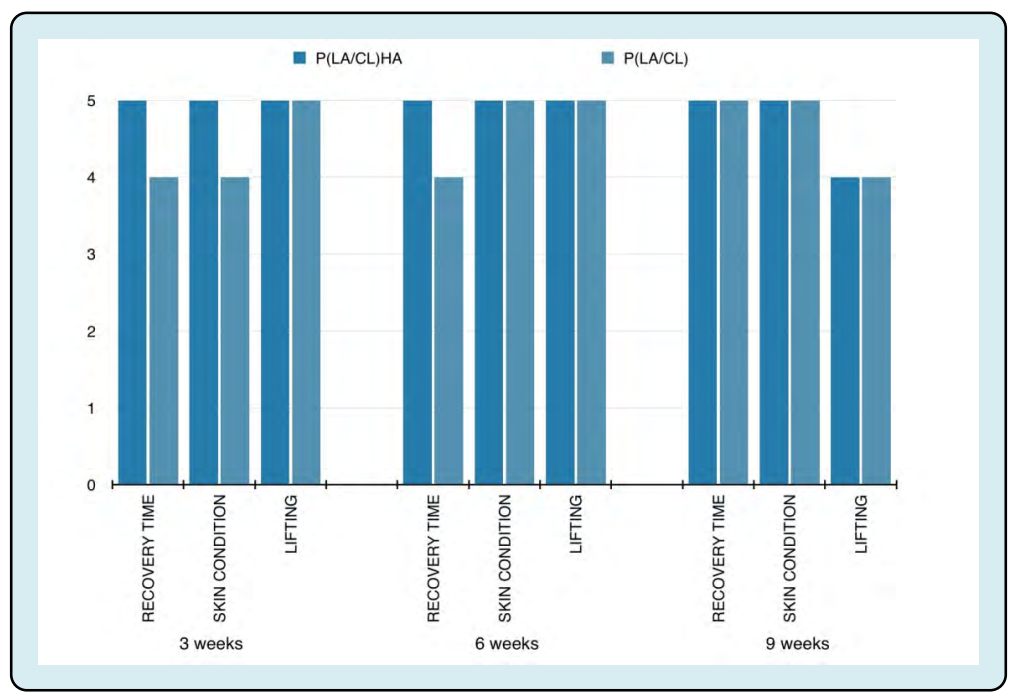

\section{Patient 5}

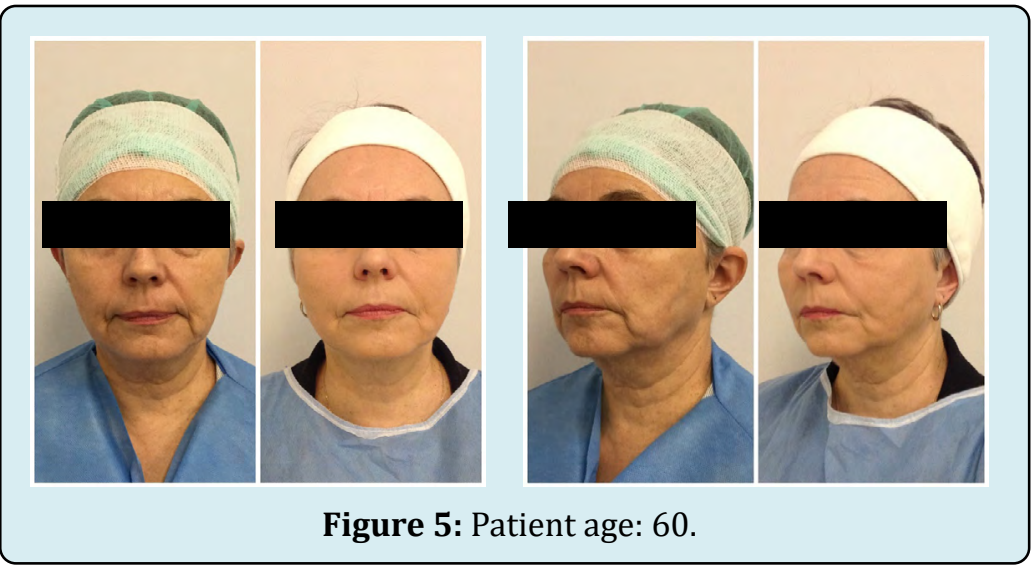

Symptoms: Loss of skin suppleness and elasticity with facial contour disorder and deep nasolabial folds and marionette furrows.

Treatment: 2 ampoules of Sculptra in deep injections (zygomatic regions, angle of the mandible, chin) and medium deep injections in the middle and lower part of the face.
P(LA/CL) and P(LA/CL) HA implantation 10 days after.

Results: Observable improvement in skin quality, better hydration, and increased skin elasticity. Significant improvement of the ovals and a reduction in marionette lines. 


\section{Patient's Assessment}

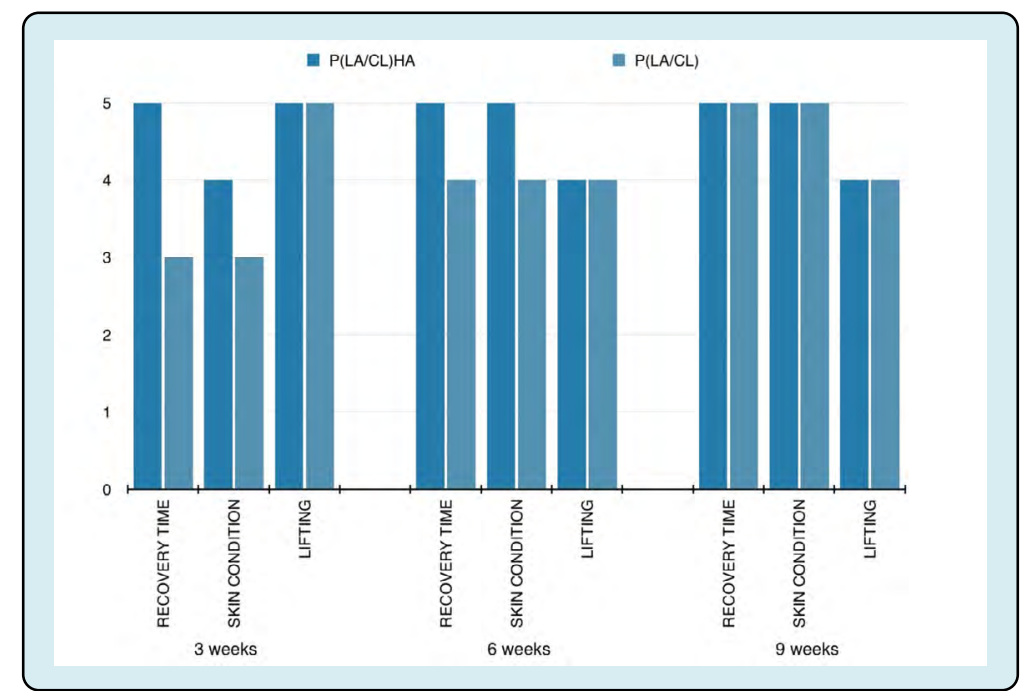

\section{Patient 6}

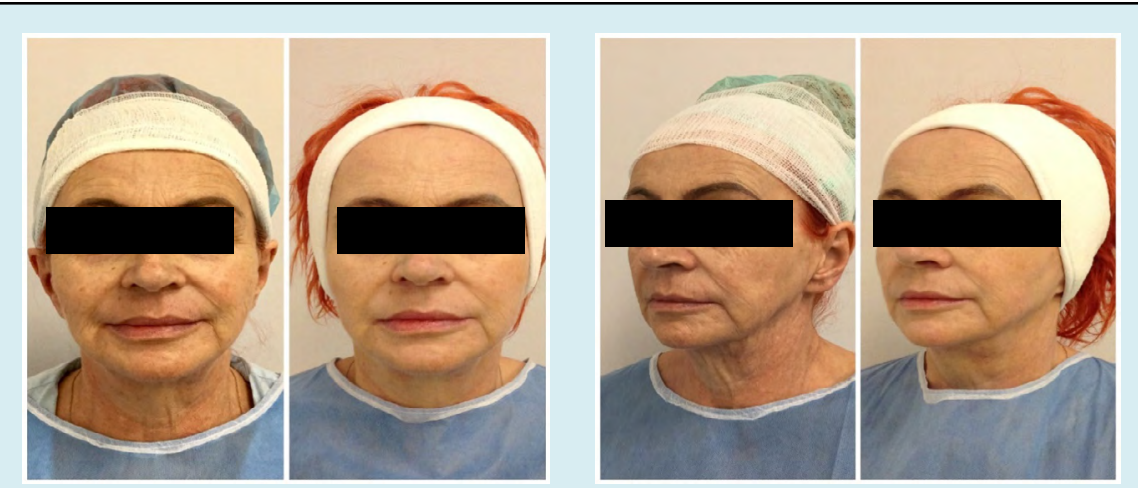

Figure 6: Patient age: 63.

Symptoms: Distinguishing features of skin elastosis with multiple wrinkles, loss of elasticity, laxity of the face and neck, and pigmentation disorder.

Treatment: 1 ampoule of Sculptra administered subcutaneously in medium deep injections. $\mathrm{P}(\mathrm{LA} / \mathrm{CL})$ and $\mathrm{P}$ P(LA/CL) HA implantation were performed after 10 days.

Results: Increased skin density and tension with visible reduction of wrinkles as well as oval improvement.

\section{Patient's Assessment}

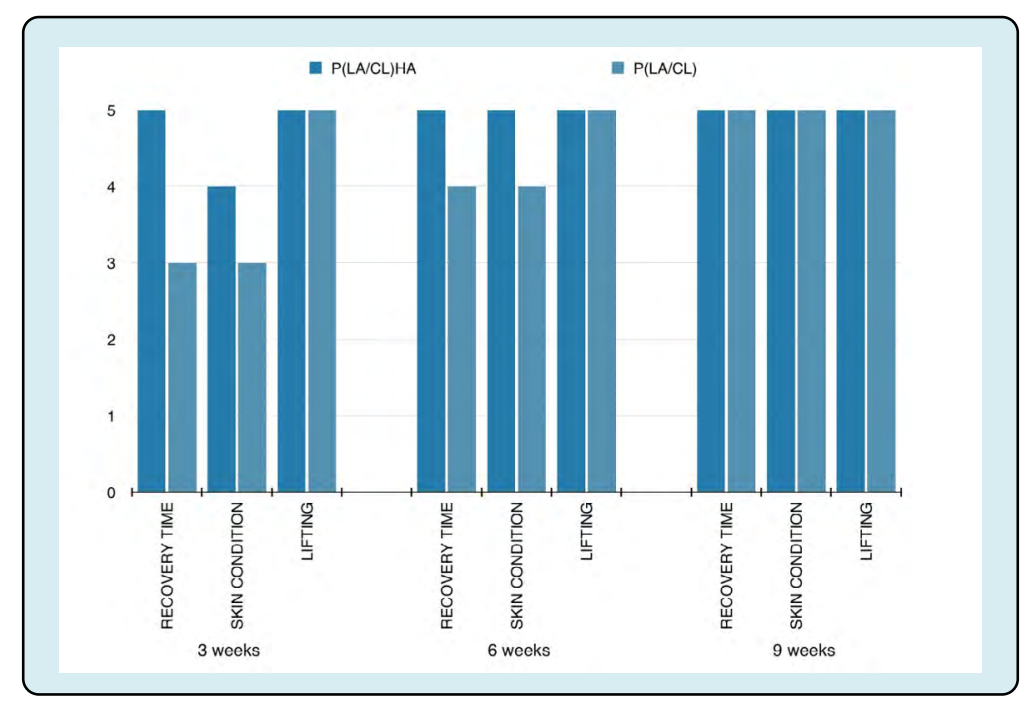




\section{Patient 7}
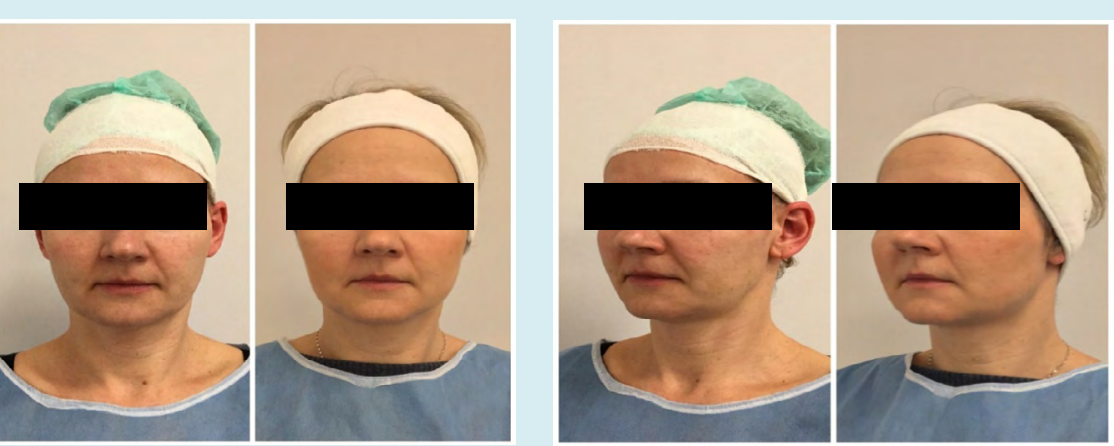

Figure 7: Patient age: 40.

Symptoms: Excessive skin dryness with decreased tissue elasticity and tension. The first visible signs of volume loss in the middle part of the face with marked nasolabial folds and marionette lines.

Treatment: $\mathrm{P}(\mathrm{LA} / \mathrm{CL})$ and $\mathrm{P}(\mathrm{LA} / \mathrm{CL}) \mathrm{HA}$ implantation with needle mesotherapy with autologous fat (nanofat).

Results: Reduction in nasolabial folds and marionette lines. Improvement in the contour and skin structure (including surface smoothing, better hydration, improvement in elasticity, and reduction in dyschromia).

\section{Patient's Assessment}

\section{Patient 8}
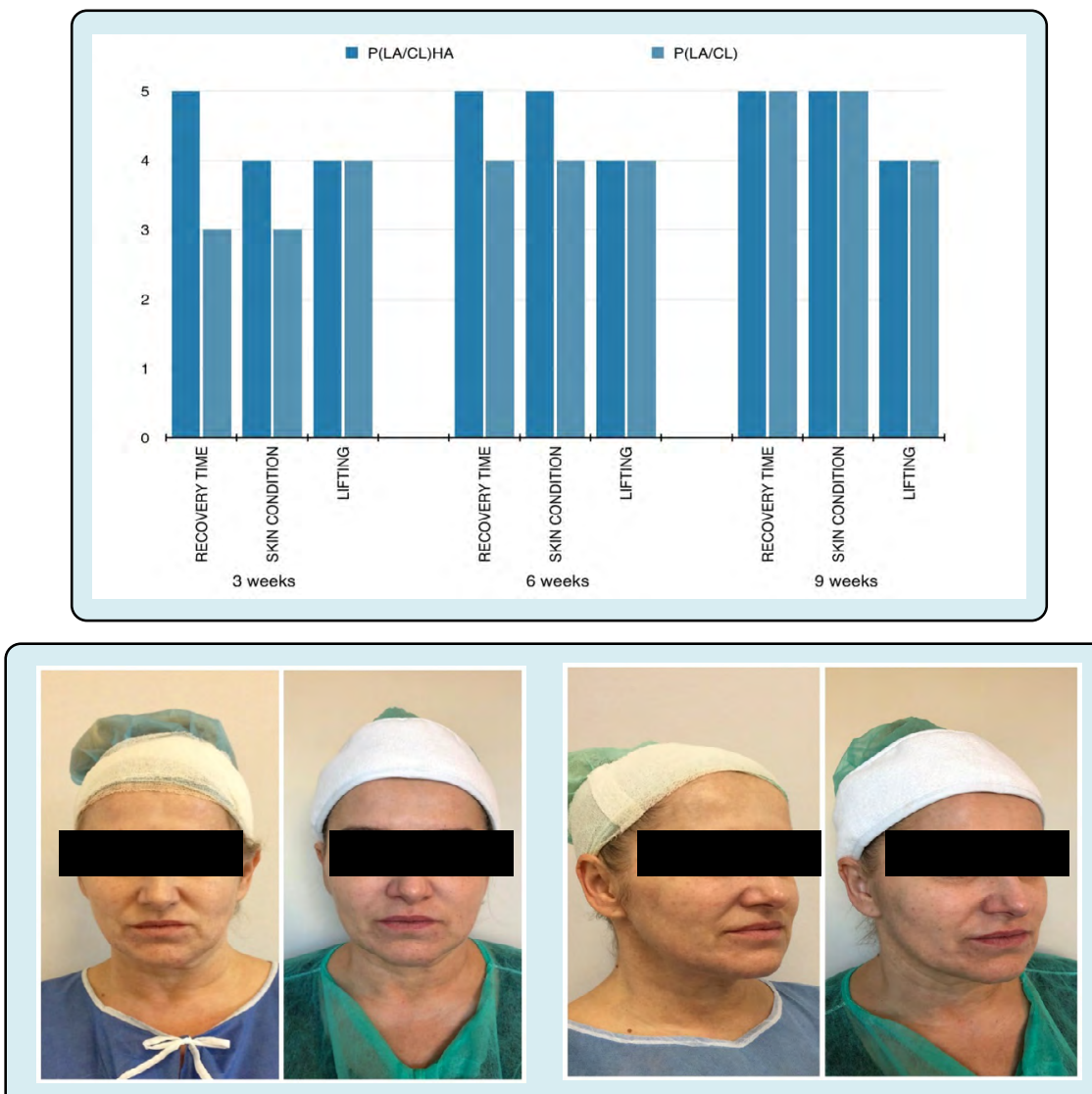

Figure 8: Patient age: 54.

Symptoms: Lipoatrophy in the middle part of the face after significant weight loss and skin laxity.

Treatment: Lipofilling (25 $\mathrm{ml}$ of autologous fat) in the temple, zygomatic area, and chin. P(LA/CL) and P(LA/CL) HA thread implantation.

Results: Lifting in the middle and lower parts of the face, 
volumetry. and skin lightening with increased tension and density.

\section{Patient's Assessment}

\section{Patient 9}
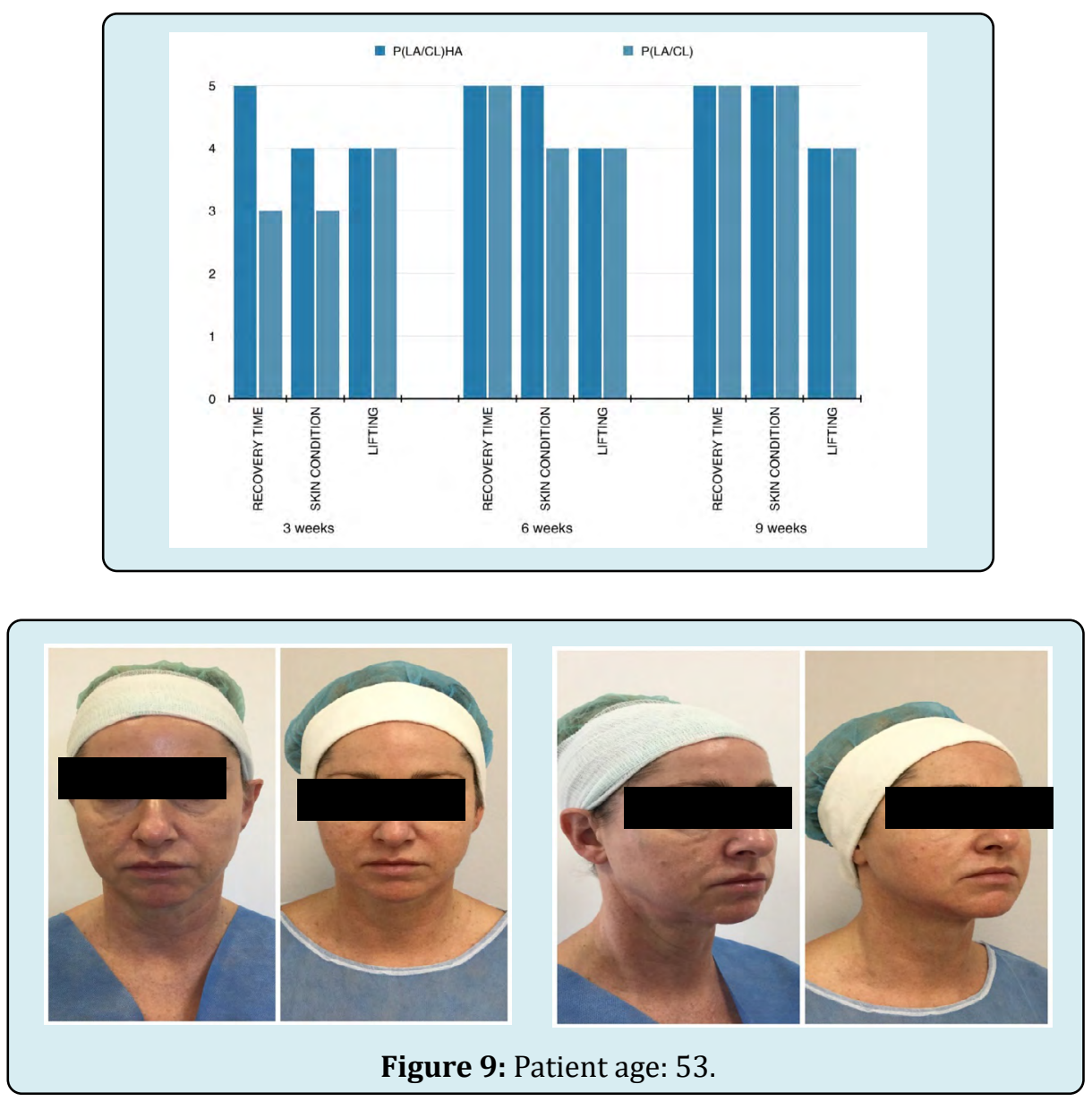

Symptoms: Lipoatrophy in the middle part of the face, clearly marked tear troughs, and an irregular jaw and chin line.

Treatment: Lipofilling in the tear troughs and middle cheek as well as implantation of $\mathrm{P}(\mathrm{LA} / \mathrm{CL}) \mathrm{HA}$ on the whole face and nonabsorbable thread Needle 2-0 in the submandibular area.

Results: Smoothing and filling of the suborbital area due to increased volume in the middle part of the cheeks and improved jaw and chin line.

\section{Patient's Assessment}

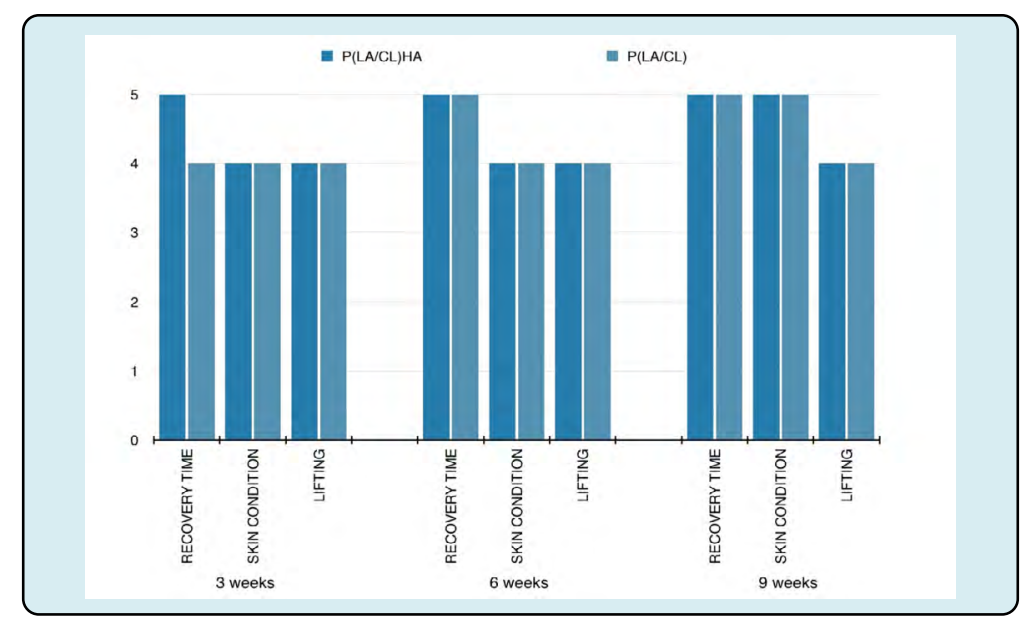




\section{Conclusion}

The results showed significant acceptance of the convalescence period, greater satisfaction with skin quality improvement, and similar lifting effects and volumetric changes.

Low traumatic treatment noticeable for both the patient and the doctor performing the procedure.

Some of the benefits of P(LA/CL) HA included a less traumatic treatment noticeable for both the patient and the doctor performing the procedure, important differences in the recovery process, noticeable especially in invasive techniques (less postoperative pain and shorter duration of edema), and earlier and more expressive signs of skin quality improvement (better tissue hydration, improvement of skin tone, and noticeable increase in skin tension). Similar tissue lifting and volume improvement were observed between P(LA/CL) and P(LA/CL) HA.

The use of hyaluronic acid significantly improved patient comfort and shortened the convalescence period. There was faster improvement in the quality of the skin within similar lifting threads.

\section{References}

1. Chao YY, Kim JW, Kim J, Ko H, Goldie K (2018) Hyperdilution of CaHA fillers for the improvement of age and hereditary volume deficits in East Asian patients. Clin Cosmet Investig Dermatol 11: 357-363.

2. Yongtrakul P, Sirithanabadeekul P, Siriphan P(2016) Thread Lift: Classification, Technique, and How to Approach to the Patient. International Scholarly and Scientific Research \& Innovation 10(12): 535-542.

3. Savoia A, Accardo C, Vannini F, Di Pasquale B, Baldi A (2014) Outcomes in thread lift for facial rejuvenation: $a$ study performed with happy lift ${ }^{T M}$ revitalizing. Dermatol
Ther: Heidelb, pp: 103-114.

4. Aston SJ, Steinbrech DS, Walden JL (2009) Aesthetic Plastic Surgery. Elsevier Health Sciences: China, pp: 5668.

5. Coleman SR, Rajiv Grover R (2006) The Anatomy of the Aging Face: Volume Loss and Changes in 3-Dimensional Topography. Aesthetic Surgery Journal 1: 4-9.

6. Tonnard P, Verpaele A, Peeters G, Hamdi M, Cornelissen $M$, et al. (2013) Nanofat Grafting: Basic Research and Clinical Applications. Plastic and Reconstructive Surgery 132(4): 1017-1026.

7. Dayan E, Chia C, Burns AJ, Theodorou S (2019) Adjustable Depth Fractional Radiofrequency Combined With Bipolar Radiofrequency: A Minimally Invasive Combination Treatment for Skin Laxity. Aesthetic Surgery Journal 39: 112-119.

8. Sulamanidze M, Sulamanidze G, Vozdvizhensky I, Sulamanidze C (2011) Avoiding Complications With Aptos Sutures. Aesthetic Surgery Journal 31(8): 863873.

9. Kang MS, Kim SH, Nam SM, Park ES (2016) Evaluation of Elastic Lift for Neck Rejuvenation. Archives of Aesthetic Plastic Surgery 22(2): 68-73.

10. Sulamanidze M, Sulamanidze G (2009) APTOS suture lifting methods: 10 years of experience. Clin Plast Surg 36(2): 281-306.

11. Beaty MM (2014) A progressive approach to neck rejuvenation. Facial Plast Surg Clin North Am 22(2): 177-190.

12. Loghem JV, Yutskovskaya YA, Philip Werschler W (2015) Calcium hydroxylapatite: over a decade of clinical experience. The Journal of Clinical and Aesthetic Dermatology 8(1): 38-49. 